\title{
Study of fusion reactions forming Cf nuclei
}

\author{
J. Khuyagbaatar ${ }^{1,2}$, D.J. Hinde ${ }^{3}$, R. du Rietz ${ }^{3}$, I. P. Carter $^{3}$, M. Dasgupta ${ }^{3}$, Ch.E. Düllmann ${ }^{1,2,4}$, M. Evers ${ }^{3}$, A. Wakhle ${ }^{3}$, E. \\ Williams $^{3}$ and A. Yakushev ${ }^{2}$ \\ ${ }^{1}$ Helmholz Institute Mainz, 55099 Mainz, Germany \\ ${ }^{2}$ GSI Helmholtzzentrum für Schwerionenforschung GmbH, 64291 Darmstadt, Germany \\ ${ }^{3}$ The Australian National University, Canberra, ACT 0200, Australia \\ ${ }^{4}$ Johannes Gutenberg-Universität Mainz, 55099 Mainz, Germany
}

\begin{abstract}
The formation of a compound nucleus in different projectile and target combinations is a powerful method for investigating the fusion process. Recently, the dominance of quasi-fission over fusion-fission has been inferred for ${ }^{34} \mathrm{~S}+{ }^{208} \mathrm{~Pb}$ in comparison to ${ }^{36} \mathrm{~S}+{ }^{206} \mathrm{~Pb}$; both reactions lead to the compound nucleus ${ }^{242} \mathrm{Cf}^{*}$. The mass and angle distributions of the fission fragments from these reactions were studied in order to further investigate the presence of quasi-fission.
\end{abstract}

\section{Introduction}

The complete fusion of two colliding nuclei forming an excited compound nucleus $(\mathrm{CN})$ is a topic of great importance in nuclear physics. In particular, the deexcitation process of such typically highly excited and rapidly rotating systems via the evaporation of neutrons (fusion-evaporation) is of interest. Many of the heaviest nuclei, with proton numbers $\mathrm{Z}>100$, have been synthesized in fusion-evaporation reactions [1,2]; such reactions remain the only means of producing these heavy nuclei as evaporation residues (ER).

In such heavy $\mathrm{CN}$, fusion-evaporation is strongly dominated by an alternate de-excitation process, namely fusion-fission. Fusion-fission is a significant deexcitation process because of two factors: first, the fission barrier is intrinsically low in such heavy $\mathrm{CN}$, and second, the high excitation and angular momentum of the $\mathrm{CN}$ produced in such reactions brings the fission barrier down even further. Therefore, ER cross sections drastically decrease with the increase in $\mathrm{CN}$ proton number. Thus, the production rate of the heavy nuclei strongly suppressed relative to the total amount of the fusion of the two colliding nuclei: as an example, the world record lowest ER cross section of $\approx 22 \mathrm{fb}\left(\mathrm{fb}=10^{-39} \mathrm{~cm}^{2}\right)$ has recently been measured for ${ }^{70} \mathrm{Zn}+{ }^{209} \mathrm{Bi}$ reaction by detecting three nuclei with $\mathrm{Z}=113$ in 553 days of beamtime [3].

The CN fusion-fission process is not solely responsible for low ER cross sections. Another mechanism, corresponding to a scenario in which the two colliding nuclei forming a two-centre quantum system fail to reach an equilibration of the all degrees of freedom leading to $\mathrm{CN}$ formation, has also been shown to lead to a reduction in ER cross sections [4]. Such non-equilibrium systems can re-separate into two fragments (quasifission) due to the strong Coulomb repulsion $\left(\sim Z_{p} \cdot Z_{t}\right)$ between the collision partners. Therefore, different projectile and target combinations leading to the formation of the same $\mathrm{CN}$ can drastically influence the ER cross sections. Thus, choosing a suitable projectiletarget combination as well as the optimum beam energy for a reaction are the most important factors for maximizing the synthesis of heavy nuclei.

Recently, an intriguing result has been observed for the formation probability of the $\mathrm{CN}\left({ }^{242} \mathrm{Cf}\right)$ in ${ }^{36} \mathrm{~S}+{ }^{206} \mathrm{~Pb}$ and ${ }^{34} \mathrm{~S}+{ }^{208} \mathrm{~Pb}$ reactions [5]. The combined data sets of the fusion-evaporation [6] and fission [5] cross section measurements provide evidence for the strong dominance of quasi-fission over fusion-fission in ${ }^{34} \mathrm{~S}+{ }^{208} \mathrm{~Pb}$ in comparison to ${ }^{36} \mathrm{~S}+{ }^{206} \mathrm{~Pb}$, in spite of the fact that both systems share the same $Z_{p} \cdot Z_{t}$ value. In the past, the presence of the quasi-fission has been predicted in two colliding nuclei with $Z_{p} \cdot Z_{t} \geq 1600$ [7]. However, more recent evidence has demonstrated the presence of quasifission for colliding systems with $Z_{p} \cdot Z_{t}$ much less than 1600 [8-11]. Thereby, the above-mentioned result for reactions leading to ${ }^{242} \mathrm{Cf}$ cannot be explained by existing models of fusion-evaporation reactions.

In general, a presence of the quasi-fission process can be defined by studying mass distributions, strong mass and angle correlations, total kinetic energy, angular distributions of the fission fragments, etc. [7,12,13]. However, such assigned can be done only in the case of the quasi-fission which occurs from a non-equilibrated system. 
Decades ago, it was demonstrated that the angular distribution of fission fragments of ${ }^{32} \mathrm{~S}+{ }^{208} \mathrm{~Pb}$ has a strong angular anisotropy that does not agree with Transition State Model predictions [14-16]. However, mass distributions of the fission fragments exhibit Gaussianlike shapes, which are typically associated with fusionfission. This observation had been explained by the presence of quasi-fission in a system that not fully equilibrated by all degrees of freedom; however, this explanation has never been confirmed by ER measurements. The dominance of quasi-fission in ${ }^{34} \mathrm{~S}+{ }^{208} \mathrm{~Pb}$ may affect the mass and angle distributions of the total fission fragments. Therefore, relatively broad mass distributions and mass-angle correlations for fission fragments from ${ }^{34} \mathrm{~S}+{ }^{208} \mathrm{~Pb}$ in comparison to ${ }^{36} \mathrm{~S}+{ }^{206} \mathrm{~Pb}$ can be treated as a sign of a higher probability of quasifission in the former system. Therefore, in this work, an experiment aimed at investigating mass and angle distributions of the fission fragments for ${ }^{34} \mathrm{~S}+{ }^{208} \mathrm{~Pb}$ and ${ }^{36} \mathrm{~S}+{ }^{206} \mathrm{~Pb}$ reactions will be presented.

\section{Experimental setup}

The experiment was carried out at the Australian National University, Canberra, Australia. Heavy ion beams were accelerated using the 14UD electrostatic accelerator. In addition to ${ }^{34} \mathrm{~S}+{ }^{208} \mathrm{~Pb}$ and ${ }^{36} \mathrm{~S}+{ }^{206} \mathrm{~Pb}$, two reference reactions, ${ }^{44} \mathrm{Ca}+{ }^{198} \mathrm{Pt}$ and ${ }^{12} \mathrm{C}+{ }^{235} \mathrm{U}$, were also studied; quasi-fission is dominant in the former reaction while fusion-fission is dominant in the latter reaction. The ${ }^{12} \mathrm{C}+{ }^{235} \mathrm{U}$ reaction leads to a different $\mathrm{CN}\left({ }^{247} \mathrm{Cf}^{*}\right)$ than the other reactions studied; however, neither ${ }^{12} \mathrm{C}+{ }^{230} \mathrm{U}$ nor ${ }^{7} \mathrm{C}+{ }^{235} \mathrm{U}$ reactions (which do lead to ${ }^{242} \mathrm{Cf}^{*}$ ) were experimentally accessible.

The ${ }^{12} \mathrm{C},{ }^{34} \mathrm{~S}$ and ${ }^{36} \mathrm{~S}$ beams were used in a short pulsed mode and ${ }^{44} \mathrm{Ca}$ beam was used in $\mathrm{DC}$ mode. Isotopically enriched ${ }^{198} \mathrm{Pt},{ }^{206} \mathrm{~Pb},{ }^{208} \mathrm{~Pb}$ and ${ }^{235} \mathrm{U}$ targets with thicknesses of about 200, 60, 170 and $20 \mu \mathrm{g} / \mathrm{cm}^{2}$ were used, respectively. Supporting thin carbon foils with typical thicknesses of (20-30) $\mathrm{ug} / \mathrm{cm}^{2}$ were used. Targets were placed at an angle of $60^{\circ}$ relative to the beam axis, with the target material layer upstream.

Coincident fission fragments were measured using the two position-sensitive multi-wire proportional counters (MWPCs) with dimensions of $28 \times 36 \mathrm{~cm}^{2}$. They were mounted in such way that the centres of the MWPCs were located at laboratory polar angles $90^{\circ}$ and $315^{\circ}$ relative to the beam axis. The distance from the centre of each MWPCs to the centre of the target was $180 \mathrm{~mm}$. Each MWPC covers scattering angle ranges of $\left(55^{\circ}-130^{\circ}\right)$ and $\left(280^{\circ}-355^{\circ}\right)$. Two small Si surface barrier detectors were used to measure elastically scattered beam for normalization of the beam intensity and target thickness. They were mounted at laboratory azimuthal and polar angles of $\pm 22.5^{\circ}$ and $0^{\circ}$, respectively, relative to the beam direction.

For the pulsed beam experiments, the velocities of fission fragments were individually determined by measuring the time-of-flight for each fragment. Only the difference in time-of-flight for each pair of coincident fission fragments was measured in the case of the DC beam; this quantity was then used to calculate fragment velocities.

Table 1. Reaction Q-values, charge products of the projectile and target proton numbers $\left(Z_{p} \cdot Z_{t}\right)$, interaction barriers according to the Bass prediction $\left(V_{\text {Bass }}\right)$ [17] and entrance channel mass asymmetries $\left(\alpha=\left(A_{t}-A_{p}\right) /\left(A_{p}+A_{t}\right)\right)$ are given.

\begin{tabular}{|c|c|c|c|c|c|}
\hline Reaction & $\mathrm{CN}$ & $\begin{array}{l}\text { Q-value } \\
(\mathrm{MeV})\end{array}$ & $\begin{array}{c}V_{\text {Bass }} \\
(\mathrm{MeV})\end{array}$ & $Z_{p} \cdot Z_{t}$ & $\alpha$ \\
\hline${ }^{44} \mathrm{Ca}+{ }^{198} \mathrm{Pt}$ & \multirow{3}{*}{${ }^{242} \mathrm{Cf}^{*}$} & -131.2 & 171.1 & 1716 & 0.64 \\
\hline${ }^{36} \mathrm{~S}+{ }^{206} \mathrm{~Pb}$ & & -113.8 & 145.1 & \multirow{2}{*}{1312} & 0.70 \\
\hline${ }^{34} \mathrm{~S}+{ }^{208} \mathrm{~Pb}$ & & -111.0 & 146.0 & & 0.72 \\
\hline${ }^{12} \mathrm{C}+{ }^{235} \mathrm{U}$ & ${ }^{247} \mathrm{Cf}^{*}$ & -25.2 & 64.7 & 552 & 0.90 \\
\hline
\end{tabular}

The measured positions and velocities of the fission fragments were then used to reconstruct the centre-ofmass angle $\left(\theta_{\text {c.m. }}\right)$ and mass ratio $\mathrm{M}_{\mathrm{R}}=\mathrm{m}_{1} /\left(\mathrm{m}_{1}+\mathrm{m}_{2}\right)$, where $\mathrm{m}_{1}$ and $\mathrm{m}_{2}$ are the two fragment masses. A detailed description of the experimental setup and data analysis can be found in $[10,11]$.

For ${ }^{12} \mathrm{C}+{ }^{235} \mathrm{U}$, only fission events originating from the ${ }^{247} \mathrm{Cf}^{*} \mathrm{CN}$ are presented. Fission events originating from the target-like nuclei were removed from the data by requiring full momentum transfer conditions for the measured fission-like events on the correlations of the centre-of-mass and azimuthal angles.

For all experiments, beam energies where chosen in order to explore reactions resulting in $\mathrm{CN}$ with similar excitation energies. However, angular momenta of the $\mathrm{CN}$ are slightly different in each reaction, due to differing mass asymmetries in the entrance channels.

\section{Experimental results}

Important entrance channel quantities for each reaction are given in Table. 1. The measured mass ratio $\left(M_{R}\right)$ and center-of-mass angle $\left(\theta_{\text {c.m. }}\right)$ of fission fragments from reactions ${ }^{44} \mathrm{Ca}+{ }^{198} \mathrm{Pt},{ }^{36} \mathrm{~S}+{ }^{206} \mathrm{~Pb},{ }^{34} \mathrm{~S}+{ }^{208} \mathrm{~Pb}$ and ${ }^{12} \mathrm{C}+{ }^{235} \mathrm{U}$ are shown in Fig. 1 as $\left(\mathrm{M}_{\mathrm{R}}, \theta_{\text {c.m. }}\right)$ plots. In Fig. 1 , projections of the selected fission events onto the $M_{R}$ axis are also shown. For both types of plots, fission fragments with mass ratios ranging from $\mathrm{M}_{\mathrm{R}}=0.25\left(0.26\right.$ for $\left.{ }^{44} \mathrm{Ca}\right)$ to $0.75\left(0.74\right.$ for $\left.{ }^{44} \mathrm{Ca}\right)$ were selected in order to exclude events from elastic and inelastic scattering. Only the fission events within $\theta_{\text {c.m. }}=\left(88^{\circ}-138^{\circ}\right)$ and $\left(90^{\circ}-132^{\circ}\right)$ were selected for $\mathrm{S}$ and $\mathrm{C}$ induced reactions, respectively, in order to exclude artificial asymmetry due to the geometric efficiency of the detectors. The measured fission events were mirrored in the case of the ${ }^{44} \mathrm{Ca}$ relative to axes $\pi-\theta_{\text {c.m. }}$ and $\left(1-\mathrm{M}_{\mathrm{R}}\right)$ in order to show the full trend of the $\left(\mathrm{M}_{\mathrm{R}}, \theta_{\text {c.m. }}\right)$ plot. Non-mirrored fission events are shown in all other cases. In the mass ratio plots, shoulders to the right and left sides of $M_{R}=0.5$ are due to elastic/inelastic scattering, which is not fully removed from the data because of reaction kinematics and experimental method (use of the DC beam, adjustment of the electronic threshold, etc.).

The MAD of fission fragments from the reaction ${ }^{44} \mathrm{Ca}+{ }^{198} \mathrm{Pt}$ shows a strong correlation between the mass 
ratio and center-of-mass angle for all measured $\mathrm{CN}$ excitation energies. This is a clear indication that the fission fragments originate from re-separation of the twocenter system during rotation. Such behavior of the fission fragments is a well-understood sign of the quasifission process $[10-13,18]$ and represents the dynamical evolution of the two captured nuclei that do not form a CN. A characteristic reaction timescale of $\leq 10^{-20} \mathrm{~s}$ has been found [18] to describe experimental MAD data in systems where quasi-fission probabilities are significant, which agrees with results obtained using the neutronclock methods [19].

The MAD for ${ }^{12} \mathrm{C}+{ }^{235} \mathrm{U}$ shows no correlation between the mass ratio and center-of-mass angle. Such behavior is typically attributed to fission of the $\mathrm{CN}$. The $\mathrm{CN}$ forms as a result of the equilibration of all the degrees of freedom in the two-center system. The measured ER cross sections of ${ }^{12} \mathrm{C}+{ }^{235} \mathrm{U}$ are on the order of $\mu$ b's [20-23], which is also consistent with the conclusion that $\mathrm{CN}$ formation is dominant in this fusion reaction. A characteristic time for the fission events from this process is, however, not possible to determine. If the two touching nuclei undergo more than one full rotation, the mass-angle distribution will not provide information on the reaction timescale, as a mass-angle correlation in the fission fragments only appears for systems that reseparate before this point. The characteristic lifetime of this fusion-fission process is expected to be relatively long $\left(>10^{-20} \mathrm{~s}\right)$ in comparison to quasi-fission. The corresponding reaction time for fusion-fission can be as long as $10^{-16} \mathrm{~s}[18,19]$. This time is estimated from the neutron-induced fission of the heavy nuclei, which similarly can also be populated in heavy $\mathrm{CN}$ after the evaporation of the neutrons [24].

Corresponding $M_{R}$ distributions of the fission fragments originating from fusion-fission and quasifission in ${ }^{12} \mathrm{C}+{ }^{235} \mathrm{U}$ and ${ }^{44} \mathrm{Ca}+{ }^{198} \mathrm{Pt}$, respectively, are symmetrically distributed around $\mathrm{M}_{\mathrm{R}}=0.5$. Widths of the $\mathrm{M}_{\mathrm{R}}$ distributions for ${ }^{44} \mathrm{Ca}+{ }^{198} \mathrm{Pt}$ become broader due to the summing of different fission fragment mass asymmetries at different center-of-mass angles as seen from the MAD spectra. In the case of ${ }^{12} \mathrm{C}+{ }^{235} \mathrm{U}$, the widths of the $\mathrm{M}_{\mathrm{R}}$ distributions do not depend on fission fragment emission angle.

No correlations between the mass ratio and center-ofmass angle were observed for ${ }^{34} \mathrm{~S}+{ }^{208} \mathrm{~Pb}$ and ${ }^{36} \mathrm{~S}+{ }^{206} \mathrm{~Pb}$ reactions. Their MADs and MR distributions are similar at the same excitation energies of ${ }^{242} \mathrm{Cf}^{*}$. Both MADs and $\mathrm{M}_{\mathrm{R}}$ distributions of these reactions are also similar to those observed for ${ }^{12} \mathrm{C}+{ }^{235} \mathrm{U}$, in which fusion-fission is solely assigned as the origin of these fission events. Therefore, at first sight, these fission events may also be thought to originate from fusion-fission forming the ${ }^{242} \mathrm{Cf}^{*} \mathrm{CN}$.

If fusion-fission is the source of these fission events for both ${ }^{34} \mathrm{~S}+{ }^{208} \mathrm{~Pb}$ and ${ }^{36} \mathrm{~S}+{ }^{206} \mathrm{~Pb}$ reactions, then the ER cross sections of these two reactions should be similar, following the $\mathrm{CN}$ hypothesis of N. Bohr [25] at excitation energies above the Bass barrier. His hypothesis states that a $\mathrm{CN}$ formed at the same excitation energy, with the same angular momentum, does not remember how it was formed. Bohr's hypothesis was originally postulated to explain the results of neutron capture reactions at low excitation energies $(<20 \mathrm{MeV})$; however, it has also been shown to be consistent with observations of light particleinduced reactions. The use of this hypothesis allows for a simplification of the theoretical description of the fusionevaporation reaction, separating it into two stages: capture and $\mathrm{CN}$-decay. Each stage can then be described independently. However, the ER cross-sections of ${ }^{34} \mathrm{~S}+{ }^{208} \mathrm{~Pb}$ are drastically lower than for ${ }^{36} \mathrm{~S}+{ }^{206} \mathrm{~Pb}$ over a wide range of the excitation energies (20-40 MeV) [5].

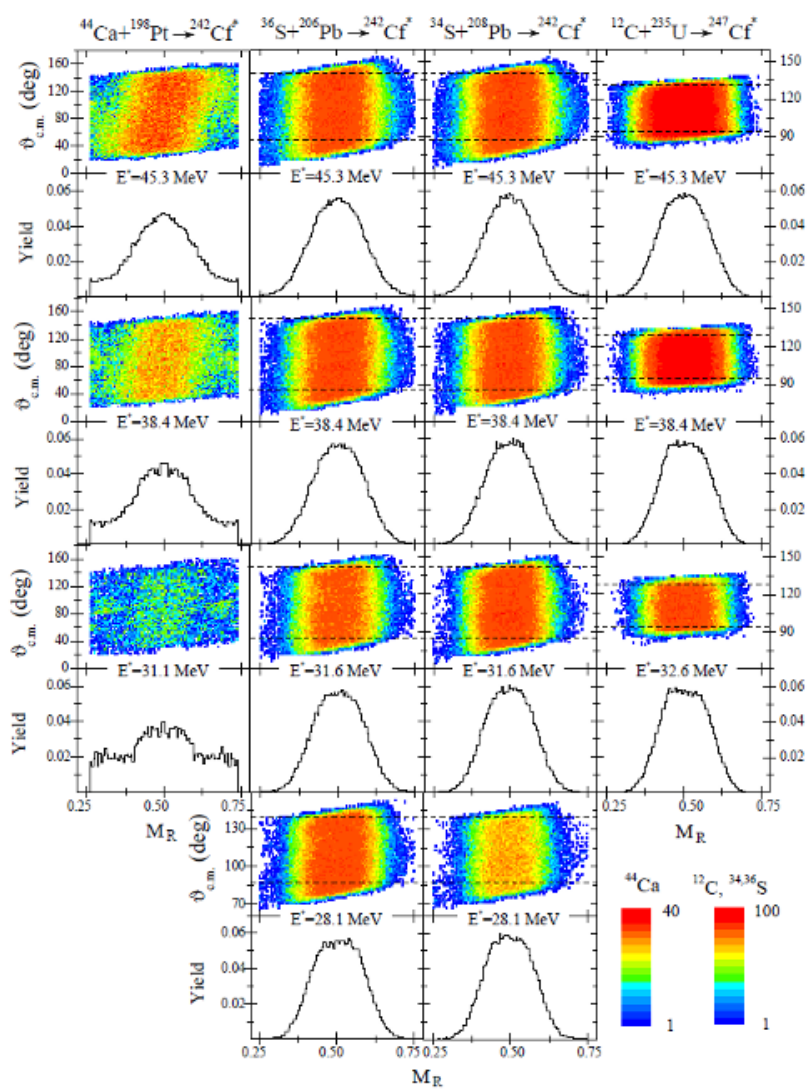

Figure 1. The measured mass ratio and center-of-mass angle distributions of fission events from the reactions ${ }^{44} \mathrm{Ca}+{ }^{198} \mathrm{Pt}$, ${ }^{36} \mathrm{~S}+{ }^{206} \mathrm{~Pb},{ }^{34} \mathrm{~S}+{ }^{208} \mathrm{~Pb}$ and ${ }^{12} \mathrm{C}+{ }^{235} \mathrm{U}$ are shown in $\mathrm{M}_{\mathrm{R}}$ and $\theta_{\text {c.m }}$ plane. Below the each MAD plots the projection of the MAD on a $M_{R}$ axis is shown. The excitation energies of the ${ }^{242} \mathrm{Cf}^{*}$ and ${ }^{247} \mathrm{Cf}^{*}$ are also given. Dashed lines show the selected fission events projected on $M_{R}$ axis. See text for details.

To reconcile these conflicting pieces of experimental evidence, one can attribute the dominant fission events from ${ }^{34} \mathrm{~S}+{ }^{208} \mathrm{~Pb}$ to a relatively long-lived quasi-fission process that does not lead to $\mathrm{CN}$ formation. This kind of quasi-fission should occur after the equilibration of the two-centre system, but before the formation of $\mathrm{CN}$. Evidence of this kind of quasi-fission has been found in the ${ }^{32} \mathrm{~S}+{ }^{208} \mathrm{~Pb}$ reaction through measurements of the angular anisotropy of the symmetrically distributed fission fragments. However, this measurement has never been confirmed by ER measurements. Therefore, we suggest a "new" type of quasi-fission, which occurs during a transition stage in which the partially equilibrated quantum system dynamically moves towards the formation of a CN. Such a transition stage has never been taken into account in heavy ion induced fusionevaporation reaction theories. The time-scale of these 
processes is expected to be within the $10^{-20} \mathrm{~s}<\tau(\mathrm{QF})<10^{-16}$ s range.

In examining the $M_{R}$ distributions of all studied reactions, as shown in Fig. 1, more curious behavior is found. For ${ }^{12} \mathrm{C}+{ }^{235} \mathrm{U}, \mathrm{M}_{\mathrm{R}}$ distributions are expected to have a Gaussian shape, as predicted by the Rotating Liquid Drop Model (RLMD) of CN-fission [24]. However, measured $\mathrm{M}_{\mathrm{R}}$ distributions of ${ }^{12} \mathrm{C}+{ }^{235} \mathrm{U}$ exhibit flat-top shapes at all measured excitation energies (see Fig. 1). This non-Gaussian character is particularly visible at low excitation energies. In the case of $\mathrm{S}$ induced reactions at high excitation energies, the $M_{R}$ distributions all have Gaussian shapes. However, at the lowest beam energies, $M_{R}$ distributions are also nonGaussian.

To explore the evolution of $M_{R}$ distributions as a function of excitation energy, the mirrored fission fragments were fit with Gaussian functions. The $M_{R}$ distributions of ${ }^{12} \mathrm{C}+{ }^{235} \mathrm{U}$ and both $\mathrm{S}$ induced reactions, together with fitted Gaussians, are shown in Fig. 2 and Fig 3, respectively. In ${ }^{12} \mathrm{C}+{ }^{235} \mathrm{U}$, it is clear that the $\mathrm{M}_{R}$ distributions have a non-Gaussian shape, and are best represented as flat-top distributions. The same flat-topped distributions are visible at excitation energies around 28 and $32 \mathrm{MeV}$ in both $\mathrm{S}$ induced reactions. At the higher excitation energies, around 38 and $52 \mathrm{MeV}, \mathrm{M}_{\mathrm{R}}$ distributions of both reactions are well fit by Gaussians, which indicates that symmetric $\mathrm{CN}$-fission is primarily responsible for these fissions.

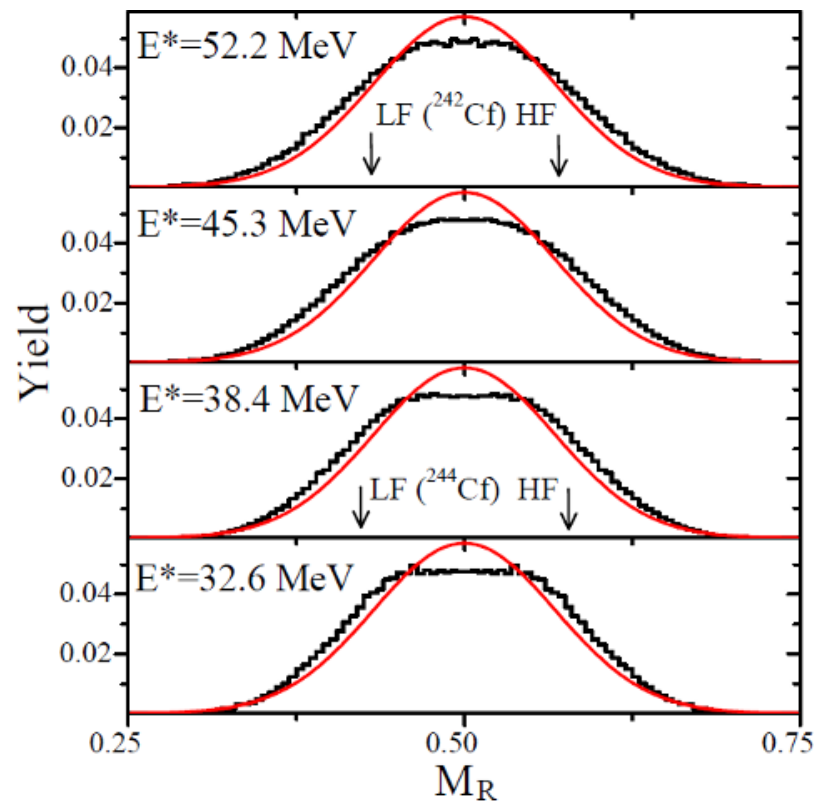

Figure 2. The $M_{R}$ distributions of the fission fragments from ${ }^{247} \mathrm{C} f^{*}$ formed in ${ }^{12} \mathrm{C}+{ }^{235} \mathrm{U}$ at different excitation energies. Fitted Gaussians are also shown by solid lines. Arrows mark the positions of heavy (HF) and light (LF) mass fission fragments of ${ }^{242} \mathrm{Cf}$ and ${ }^{244} \mathrm{Cf}$.

Root squared standard deviations $\left(\sigma_{\mathrm{MR}}\right)$ of the fitted Gaussians are plotted as a function of excitation energies of the $\mathrm{CN}$ in Fig. 4 (upper panel). Standard deviations for ${ }^{12} \mathrm{C}+{ }^{235} \mathrm{U}$ are lower than those for $\mathrm{S}+\mathrm{Pb}$, which is attributed to the relatively low angular momentum (see below) of ${ }^{247} \mathrm{Cf}^{*}$ compared to ${ }^{242} \mathrm{Cf}^{*}$. For all reactions, a smooth decreasing (linear) trend in the $\left(\sigma_{\mathrm{MR}}\right)^{2}$ values is observed as excitation energies (temperature) decrease. Such a linear dependence of the $\left(\sigma_{\mathrm{MR}}\right)^{2}$ values on the temperature of the $\mathrm{CN}$ conforms with the prediction of the RLDM. However, at low excitation energies, the smooth linear dependence of $\left(\sigma_{M R}\right)^{2}$ values on the temperature of the $\mathrm{CN}$ is broken for both S-induced reactions.

The $\left(\mathrm{RMS} / \sigma_{\mathrm{MR}}\right)_{\text {nor. }}$ ratios between the standard deviations of the data (Root Mean Square-RMS) and the fitted Gaussians are also shown in Fig. 4 (lower panel). These ratios represent the strength of the agreement between the experimental and assumed shapes of the $M_{R}$ distributions. The value 1 means that the experimental $\mathrm{M}_{\mathrm{R}}$ distribution has a Gaussian shape as predicted by the RLDM of the CN-fission. The ratios of the experimental data $\left(\left(\mathrm{RMS} / \sigma_{\mathrm{MR}}\right)_{\text {exp. }}\right)$ were normalized to the ratios of the randomly generated Gaussian events $\left(\left(\mathrm{RMS} / \sigma_{\mathrm{MR}}\right)_{\text {gaus. }}\right)$ in order to properly account for the artificial reduction of RMS values within the selected $M_{R}=(0.25-0.75)$ range.

In the case of the $S$ induced reactions, the $\left(\mathrm{RMS} / \sigma_{\mathrm{MR}}\right)_{\text {nor. }}$ values are equal to 1 within the error bars above the excitation energy $38 \mathrm{MeV}$ (See Fig. 3). This demonstrates that the $\mathrm{M}_{\mathrm{R}}$ distributions are well-described by Gaussians and are consistent with the RLDM predictions. However, at lower excitation energies, ratios are more than 1 , which quantitatively demonstrates that the $\mathrm{M}_{\mathrm{R}}$ distributions are not described by Gaussians (See Fig. 3). These non-Gaussian $M_{R}$ distributions are the reason for the breaking of the linear dependence between the $\left(\sigma_{\mathrm{MR}}\right)^{2}$ and temperature.

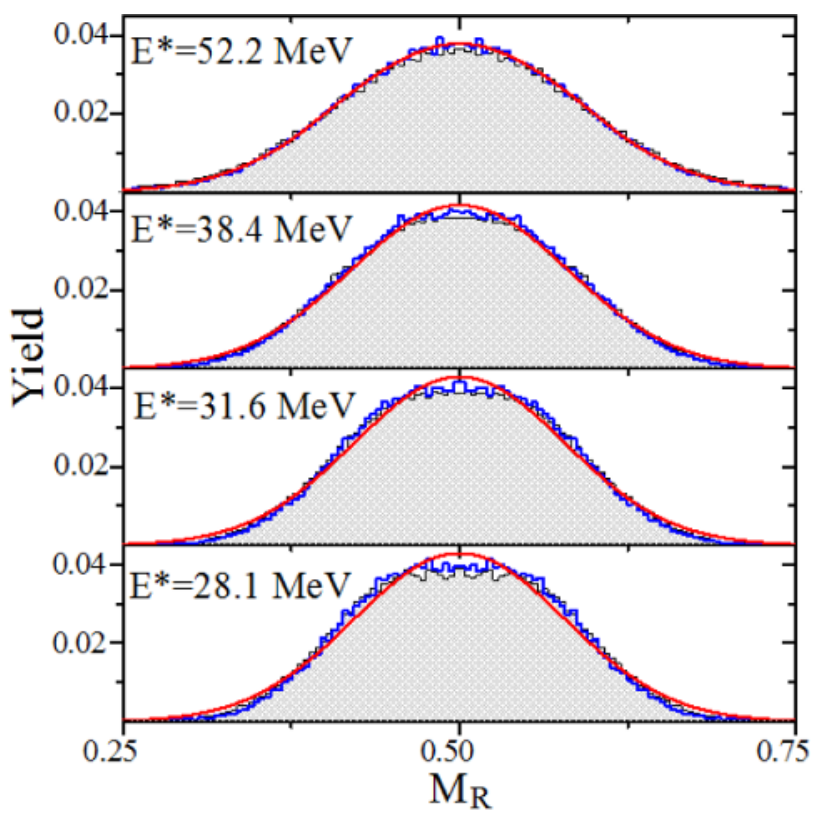

Figure 3. The experimental $M_{R}$ (mirrored) distributions of the ${ }^{242} \mathrm{Cf}^{*}$ formed in ${ }^{34} \mathrm{~S}+{ }^{208} \mathrm{~Pb}$ (filled-line) and ${ }^{36} \mathrm{~S}+{ }^{206} \mathrm{~Pb}$ (thickline) at different excitation energies. Fitted Gaussians of the $\mathrm{M}_{R}$ distributions from the ${ }^{34} \mathrm{~S}+{ }^{208} \mathrm{~Pb}$ are shown by the solid curves.

Large deviations of $\left(\mathrm{RMS} / \sigma_{\mathrm{MR}}\right)_{\text {nor }}$ from 1 were deduced for the $\mathrm{M}_{\mathrm{R}}$ distributions of ${ }^{12} \mathrm{C}+{ }^{235} \mathrm{U}$, as expected from Fig. 2. 


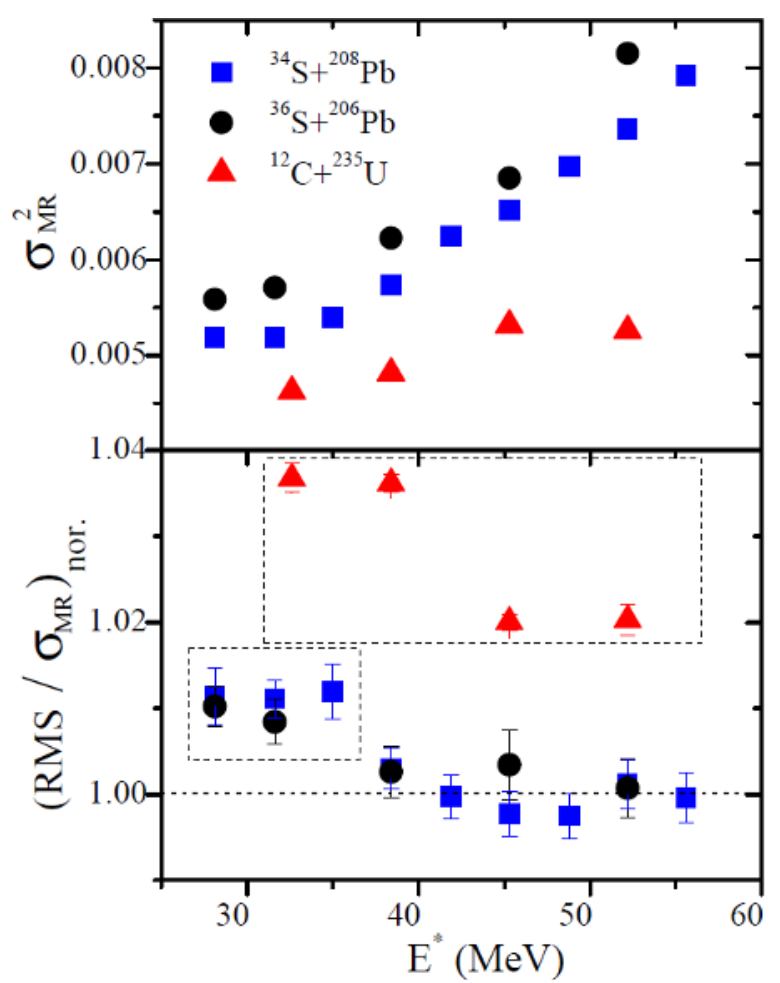

Figure 4. Root squared variances $\left(\sigma_{\mathrm{MR}}\right)$ of the Gaussian fit and ratio $\left(\mathrm{RMS} / \sigma_{\mathrm{MR}}\right)_{\text {nor. }}$ of the $\mathrm{M}_{\mathrm{R}}$ distributions for the three reactions are shown as a function of excitation energy (upper part). Uncertainties of values are smaller than the size of the symbols. The dotted line indicates the value 1 , which shows the best agreement between the $M_{R}$ distribution and the Gaussian fit. Dashed line boxes emphasize the $\mathrm{M}_{\mathrm{R}}$ distributions that have non-Gaussian shapes.

Flat-topped $\mathrm{M}_{\mathrm{R}}$ distributions in ${ }^{12} \mathrm{C}+{ }^{235} \mathrm{U}$ cannot be due to the presence of quasi-fission and are expected to originate from fusion-fission. In this case, the $\mathrm{CN}$-fission occurs via different paths: (i) mass-symmetrically distributed fission fragments, as predicted by the RLDM, and (ii) mass-asymmetrically distributed fission fragments. The latter process may originate from low energy $\mathrm{CN}$-fission, which is possible only in late chance fissions from the excited CN. It is well known that fission from ground and excited states of the heavy nuclei is strongly influenced by shell structure. Low energy fissions of the neutron-deficient $\mathrm{Cf}$ nuclei have asymmetric mass distributions, which will easily produce a flat-topped distribution mixed with symmetric $\mathrm{CN}$ fission. As an example, low energy fissions of the neutron-deficient ${ }^{242} \mathrm{Cf}^{*}$ and ${ }^{244} \mathrm{Cf}^{*}$ are experimentally known from the electron capture decays of ${ }^{242}$ Es and ${ }^{244} \mathrm{Es}$ [26,27]. Corresponding $M_{R}$ values of the fission fragments from these nuclei are marked in Fig. 2.

An assumption about the presence of late chance CNfissions can be easily made based on observed ERs in neutron evaporation channels [20-23]. It is interesting to note that the $\mathrm{xn}$ ER excitation functions of such asymmetric reactions (for example, ${ }^{12} \mathrm{C}+{ }^{233-236,238} \mathrm{U}[20-$ 23]) yield maxima for the evaporation of 4-6 neutrons, which corresponds to roughly the same excitation energy region (from 35 to $55 \mathrm{MeV}$ ) in which flat-top distributions are observed in this work. In a late chance fission process, de-excitation of the $\mathrm{CN}$ occurs through different stages at each "newly" formed $\mathrm{CN}$, with the reduced atomic mass number and reduced excitation energy enhancing survival against fission. Such a process may continue down to excitation energies that are above both the neutron binding energy and fission barrier of the "last" CN. In the case of neutron-deficient Cf nuclei, the neutron binding energy and fission barriers are below 9 $\mathrm{MeV}$. Thus, surviving $\mathrm{CN}$ down to around this energy will be the main sources of the observed xn ERs.

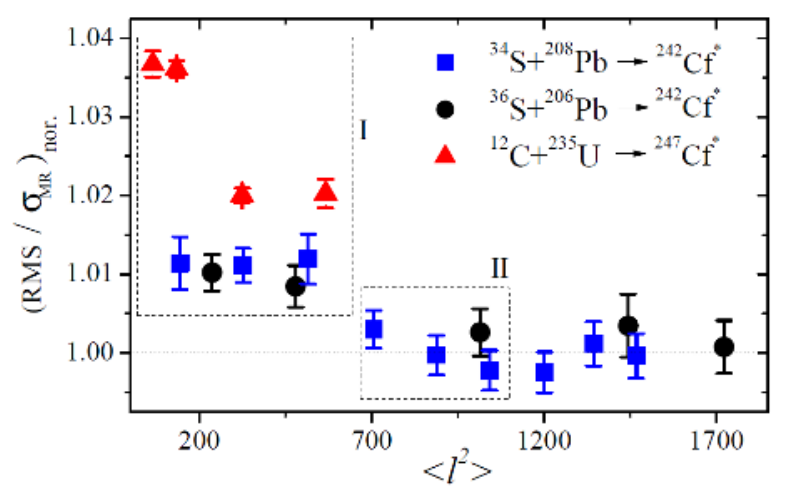

Figure 5. Ratio (RMS/ $\left.\sigma_{M R}\right)_{\text {nor. }}$ of the $M_{R}$ distributions for the three reactions are shown as a function of root mean squared angular momentum.

The flat-top becomes more pronounced with decreasing $\mathrm{CN}$ excitation energy and also angular momentum. This property can easily be explained as a result of the increase in the total fission barrier of the initial $\mathrm{CN}$ at lower excitation energies and angular momenta. Thus, with an increase in the total fission barrier, the survival probability of the $\mathrm{CN}$ against early chance fissions is increased.

The origin of the flat-topped $\mathrm{M}_{\mathrm{R}}$ distributions in ${ }^{34} \mathrm{~S}+{ }^{208} \mathrm{~Pb}$ and ${ }^{36} \mathrm{~S}+{ }^{206} \mathrm{~Pb}$ may be the same as that suggested above for the ${ }^{12} \mathrm{C}+{ }^{235} \mathrm{U}$. However, flat-topped $\mathrm{M}_{\mathrm{R}}$ distributions for ${ }^{34} \mathrm{~S}+{ }^{208} \mathrm{~Pb}$ and ${ }^{36} \mathrm{~S}+{ }^{206} \mathrm{~Pb}$ appear at relatively low excitation energies compared to those observed in ${ }^{12} \mathrm{C}+{ }^{235} \mathrm{U}$. This difference in excitation energies can be due to the different $\mathrm{CN}$ formed $\left({ }^{242} \mathrm{Cf}^{*}\right.$ and ${ }^{247} \mathrm{Cf}^{*}$ formed in $\mathrm{S}+\mathrm{Pb}$ and ${ }^{12} \mathrm{C}+{ }^{235} \mathrm{U}$ reactions, respectively). The nucleus ${ }^{247} \mathrm{Cf}$ should have stronger shell effects compared to the neutron-deficient ${ }^{242} \mathrm{Cf}$ because of neutron excess close to $\mathrm{N}=152$. However, the most probable reason for the difference is likely to be the different angular momenta of these CNs.

The same $\left(\mathrm{RMS} / \sigma_{\mathrm{MR}}\right)_{\text {nor. }}$ values shown in Fig. 4 are drawn in Fig. 5 as a function of the root mean squared angular momentum of the $\mathrm{CN}$. The average angular momentum of the $\mathrm{CN}$ formed in each reaction was calculated in the coupled-channels code CCFULL, which takes into account the couplings of low-lying excited states and deformations [28]. As Fig. 5 shows, plotting $\left(\mathrm{RMS} / \sigma_{\mathrm{MR}}\right)_{\text {nor. }}$ values with respect to root mean squared angular momentum reveals a more systematic picture than the same data plotted with respect to excitation energies. Only flat-top distributions were observed in the region marked by (I) in Fig. 5 for all three reactions. A marked region (II) seems to be an intermediate; above this region, the $M_{R}$ distributions are best described as Gaussian. The much higher angular momenta of ${ }^{242} \mathrm{Cf}^{*}$ 
compared to ${ }^{247} \mathrm{Cf}^{*}$ at high excitation energies ( $>38 \mathrm{MeV}$ ) (see Fig. 4 and Fig. 5) decrease the total fission barrier (at least the liquid drop part of the fission barrier). Thus, early chances $\mathrm{CN}$-fissions are more probable in comparison to ${ }^{12} \mathrm{C}+{ }^{235} \mathrm{U}$ at excitation energies above 38 $\mathrm{MeV}$. Again, it is very important to note that the xn ER excitation functions of the $\mathrm{S}+\mathrm{Pb}$ reactions yield maxima for the 2-3 neutron evaporations (roughly from 20 to 35 $\mathrm{MeV}[5,6])$ where the flat-top becomes visible.

As suggested above, a "new" type of quasi-fission with a relatively long reaction timescale must be dominant in ${ }^{34} \mathrm{~S}+{ }^{208} \mathrm{~Pb}$ in comparison to ${ }^{36} \mathrm{~S}+{ }^{206} \mathrm{~Pb}$. Thus, fission events contributing to $\mathrm{M}_{\mathrm{R}}$ distributions for ${ }^{34} \mathrm{~S}+{ }^{208} \mathrm{~Pb}$ must correspond at least in part to fission events originating from this quasi-fission process. However, the $M_{R}$ distributions are the same for both reactions (Fig. 3) at all measured excitation energies. The only explanation for this uniform behavior is that the long-lived quasi-fission process is somehow very similar to the fusion-fission process. An equilibrated and most probably still deformed system can follow along the potential energy surface of the $\mathrm{CN}$, but may not overcome the saddle point of the inner fission barrier of the $\mathrm{CN}$. At the same time, this system also cannot reseparate, perhaps because there are no asymmetric potential energy minima. Therefore, at the end, this system will reach the ground state potential energy paths, meaning that the fission of such a system must follow the fission properties of the CN. Therefore, flat-top distributions from the ${ }^{34} \mathrm{~S}+{ }^{208} \mathrm{~Pb}$ reactions can primarily originate from asymmetrically and symmetrically massdistributed quasi-fission processes.

\section{Summary and conclusions}

Fission fragments from the ${ }^{12} \mathrm{C}+{ }^{235} \mathrm{U},{ }^{34} \mathrm{~S}+{ }^{208} \mathrm{~Pb},{ }^{36} \mathrm{~S}+{ }^{206} \mathrm{~Pb}$ and ${ }^{44} \mathrm{Ca}+{ }^{198} \mathrm{Pt}$ reactions were measured at the beam energies of around/above the Bass barriers.

Strong mass-angle correlations were observed for the ${ }^{44} \mathrm{Ca}+{ }^{198} \mathrm{Pt}$ reaction at excitation energies $(30-38) \mathrm{MeV}$ of ${ }^{242} \mathrm{Cf}^{*}$. This correlation was attributed to quasi-fission.

No mass-angle correlations were observed for the fission fragments from ${ }^{12} \mathrm{C}+{ }^{235} \mathrm{U}$. These fission events were attributed to fusion-fission.

No mass-angle correlations were observed for ${ }^{34} \mathrm{~S}+{ }^{208} \mathrm{~Pb}$ and ${ }^{36} \mathrm{~S}+{ }^{206} \mathrm{~Pb}$.

A relatively long-lived $\left(10^{-20}-10^{-16} \mathrm{~s}\right)$ "new" type of quasi-fission process is suggested to be the origin of most of the fission events observed for ${ }^{34} \mathrm{~S}+{ }^{208} \mathrm{~Pb}$.

Surprisingly, non-Gaussian mass ratio distributions were observed for ${ }^{12} \mathrm{C}+{ }^{235} \mathrm{U}$ at all observed excitation energies $(32-52 \mathrm{MeV})$ of $\mathrm{CN}\left({ }^{247} \mathrm{Cf}^{*}\right)$, which disagree with the symmetric CN-fission predicted in RLMD. Such flat-topped distributions were also observed for ${ }^{34} \mathrm{~S}+{ }^{208} \mathrm{~Pb}$ and ${ }^{36} \mathrm{~S}+{ }^{206} \mathrm{~Pb}$, which lead to ${ }^{242} \mathrm{Cf}^{*}$; however, these observations were at lower excitation energies $(<38$ $\mathrm{MeV}$ ). Flat-top distributions are attributed to the contribution of low-energy $\mathrm{CN}$ fission originating from the late chance fissions of $\mathrm{CN}$.

A comparison of the mass ratio distributions of the fission fragments from ${ }^{34} \mathrm{~S}+{ }^{208} \mathrm{~Pb}$ and ${ }^{36} \mathrm{~S}+{ }^{206} \mathrm{~Pb}$ at the same excitation energies does not show a presence of "classical" quasi-fission in ${ }^{34} \mathrm{~S}+{ }^{208} \mathrm{~Pb}$. Moreover, the observations of similar mass ratio distributions at excitation energies below $38 \mathrm{MeV}$ suggests that a proposed long-lived quasi-fission outcome could produce both symmetric and asymmetric mass distributions of fission fragments, similar in many respects to those observed for $\mathrm{CN}$-fissions.

This work has shown that fission fragment measurements alone cannot always distinguish between events originating from fusion-fission (CN-fission) and quasi-fission. Only measurements of ER cross sections for reactions forming the same $\mathrm{CN}$ will be sensitive enough to determine the presence of each process. Both theoretical and experimental studies involving all possible observable variables are needed in order to understand the dynamics of nuclear fusion.

\section{Acknowledgements}

We are indebted to Dr. N. Lobanov and the staff of the ANU Heavy Ion Accelerator Facilty for their assistance with accelerator operations. One of us (J. K.) would like to thank the Australian National University for the financial support during his stay at ANU.

\section{References}

1. S. Hofmann and G. Münzenberg, Rev. Mod. Phys. 72, 733 (2000).

2. Yu.Ts. Oganessian, J. Phys. G 34, R165 (2008).

3. K. Morita et al., J. Phys. Soc. J. 81, 103201 (2012).

4. D.J. Hinde et al., Phys. Rev. Lett. 89, 282701 (2002).

5. J. Khuyagbaatar et al., Phys. Rev. C 86, 064602 (2012).

6. J. Khuyagbaatar et al., Eur. Phys. J. A 46, 59 (2010).

7. W.J. Swiatecki, Phys. Scr. 24, 113 (1981).

8. A.C. Berriman et al., Nature (London) 413, 144 (2001).

9. A.Yu. Chizhov et al., Phys. Rev. C. 67, 011603(R) (2003).

10. R. Rafiei et al., Phys. Rev. C. 77, 024606 (2008).

11. R.G. Thomas et al., Phys. Rev. C. 77, 034610 (2008).

12. R. Bock et al., Nucl. Phys. A. 388, 334 (1982).

13. J. Toke et al., Nucl. Phys. A. 440, 327 (1985).

14. M.B. Tsang et al., Phys. Rev. C. 28, 747 (1983).

15. B. B. Back et al., Phys. Rev. Lett. 50, 818 (1983).

16. B. B. Back et al., Phys. Rev. C. 32, 195 (1985).

17. R. Bass, Phys. Rev. Lett. 39, 265 (1977).

18. R. du Rietz et al., Phys. Rev. Lett. 106, 052701 (2011).

19. D.J. Hinde et al., Phys. Rev. C. 45, 1229 (1992).

20. T. Sikkeland et al., Phys. Rev. 169, 1000 (1968).

21. R.J. Silva et al., Phys. Rev. C 2, 1948 (1970).

22. T. Ohtsuki et al., CYRIC annual report-2004, 105 (2004).

23. K. Takamiya et al., CYRIC annual report-2005, 99 (2005).

24. R Vandenbosch and J. R. Huizenga, Nuclear Fission, Academic, New York, (1973).

25. N. Bohr, Nature 137, 344 (1936).

26. D.A. Shaughnessy et al., Phys. Rev. C 61, 044609 (2000).

27. D.A. Shaughnessy et al., Phys. Rev. C 65, 024612 (2002).

28. K. Hagino et al., Comp. Phys. Comm. 123, 143 (1999). 